Available online at: http://proceeding.rsfpress.com/index.php/ic-smart/index

Proceeding on International Conference of Science Management Art Research Technology

(IC-SMART)

Volume 1 Number 1 (2020): 137-145

\title{
Arteriovenous Fistula Stenosis Detection using Noninvasive Bilateral PPG Technique with SVM-OAA Algorithm
}

\author{
Alphin Stephanus \\ Electrical Engineering Department, Ambon State Polytechnic, Indonesia, Maluku Province, \\ Ambon. \\ E-mail address peppymusila52@gmail.com
}

\begin{abstract}
This paper presents a bilateral photoplethysmography (PPG) based noninvasive technique for monitoring arteriovenous fistula (AVF) stenosis. Toward effectively extract the features of $P P G$ and classification, a real-time processing method of feature detection and support vector machines one-against-all (SVM-OAA) were used. Preliminary findings from 22 subject tests showed that the proposed approach could be provided positive predictive value $=89.0$ $\%$ and sensitivity $=83.3 \%$ estimation of AVF stenosis.
\end{abstract}

Keywords: AVS stenosis, bilateral photoplethysmograph, SVM-OAA

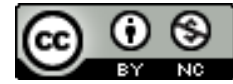

This is an open access article under the CC-BY-NC license

\section{INTRODUCTION}

Hemodialysis (HD) is one of the most frequently used treatments for patients with end-stage renal disease (ESRD) (National Kidney Foundation, 2006). In HD treatment, dialyzers are used to clean the blood from waste products. The vascular access is where a dialyzer needle is inserted into the patient's arm to obtain blood. Arteriovenous Fistula (AVF) is the most conventional type of vascular access. This AVF is created through an arm surgery to connect an artery and a vein. This connection point of arteries and veins is termed an anastomosis and is usually placed on the elbow or wrist. The fistula condition might gradually deteriorate. The venous stenosis is the most common form of this condition (Vesquez et.al, 2009).

In long-term HD treatment, inadequate venous outflow or arterial inflow is caused by access occlusion as a result of repeated puncturing. This causes a cellular deposit, chronic fibrin, limb ischemia, aneurysm, and thrombosis (National Kidney Foundation, 2006; Wu et.al, 2015).. The revision and remedied process must be carried out immediately in the event of an access occlusion. To prevent total occlusion, early detection of venous stenosis can prolong the life of the fistula. 
Non-invasive blood volume changes in the microvascular bed tissue can be sensed using PPG as an optical measurement technique. The shape of the PPG signal expresses some specific physiological information in blood changes volume, each heartbeat, respiration, thermoregulation, the activity of vasomotor (Wu et.al, 2012). PPG signal data can be retrieved using a multi-channel measurement technique placed on the great toe, ear lobes, thumbs, and index finger. The results of this measurement can then be used in pulse waveform analysis and digital signal processing (Allen, 2007). Therefore, the measurement technique using PPG is an expectant solution for detecting AVF stenosis.

Other techniques that can detect vascular stenosis clinically are angiography and Doppler ultrasound. The diameter of the arteriovenous fistula can be measured using a Doppler ultrasound. The drawback of these two techniques is that the equipment is expensive and requires advanced and experienced operators. Besides, angiography requires surgery and is invasive(Bash et.al, 2005; Tessitore et.al, 2003).

Recently, several researchers have evaluated arteriovenous fistula stenosis in various ways. The support vector machine and wavelet algorithms have been used by Vasquez at al. in 2009 to detect arteriovenous fistula stenosis via blood flow sounds (Vesquez et.al, 2009). Wang et al. used the time and frequency domain method to extract some features of the blood flow sounds of stenosed arteriovenous fistulas. Wu et al. using data on the difference between the rise time (RT) and the amplitudes (AMPs) of the Bilateral PPG signal in blood volume obtained from the left-side and right-side hands of HD treatment patients. The difference is used as a reference for comparison to the stenosis degree (DoS). When an asynchronous heartbeat on the bilateral PPG signal occurs, it means that vascular access obstruction has appeared on one side. Furthermore, Leon et al.'s study stated that the intra-flow decreases occur when vascular access that experiences stenosis is characterized by significant intra-flow decreases in the inflow and outflow [10]. The detection of AVF stenosis can be provided from many features of the PPG signal, including shape, rise time, width ratio amplitude, and pulse transit time (Wu et.al, 2015).

In this study, Noninvasive Bilateral PPG waveform feature analysis was introduced to detect AVF stenosis. PPG signal was evaluated before and after HD treatment to get its bilateral differences (left and right side) of two peaks. These result used as the feature which purposed AVF stenosis detection technique. These features contain blood volume changes in time domains. Furthermore, the SVM-OAA algorithm was used to classify three degrees of stenosis (DoS). Twenty-two patients Bilateral PPG signal data will be examined using the proposed technique.

\section{LITERATURE REVIEW}

\section{II.1. Binary SVM}

Vapnik and Cortes in their writing declare an $\mathrm{n}$ training sample: $\{\mathrm{x} 1, \mathrm{y} 1\}, \ldots,\{\mathrm{xn}, \mathrm{yn}\}$, where $\mathrm{xi} \in \mathrm{Rm}$ is an m-dimensional vector of a feature that defines the ith training sample for label class xi represented by yi $\in\{-1,1\}$. In the equation, $w T x+b=0$, a hyperplane is expressed in a state-space where $\mathrm{w} \in \mathrm{Rm}$ and $\mathrm{b}$ are a scalar. In the case of the training, samples are separated linearly, the optimal hyperplane that split two classes without error training, and the 
minimum distance of the hyperplane and training samples is maximized. The parameter pair $(\mathrm{w}, \mathrm{b})$, which is the solution of the optimization problem which corresponds to the optimal hyperplane, can be easily described in the following equation:

minimize: $L(w)=\frac{1}{2}\|w\|^{2}$

subject to: $\quad y\left(x^{T} x_{i}+b\right) \geq 1, i=1, \cdots, n$

The concept of soft margin is used in linearly nonseparable cases. The new optimization problems could be generalized to:

minimize: $\quad \mathrm{L}\left(\mathrm{w}, \xi_{\mathrm{i}}\right) \overline{\mathbf{2}}^{-}\|w\|^{2}+C \sum_{\mathrm{i}=1}^{\mathrm{n}} \overline{\mathrm{i}}$

subject to: $\left.y\left(x^{\mathrm{T}} \mathrm{x}_{\mathrm{i}}\right)+\mathrm{b}\right) \geq 1-\xi_{\mathrm{i}}, \mathrm{i}=1, \cdots \mathrm{n}$,

The slack variable, which is denoted by $\xi \mathrm{i}$ as in Figure 1, is related to the soft margin and to balance the error of training, and the margin is used tuning parameter $\mathrm{C}$. The optimization problem is shown in equations

(1) and (2) could be answered by introducing the multipliers of Lagrange $\alpha \mathrm{i}$ that transform them into problems of quadratic programming.

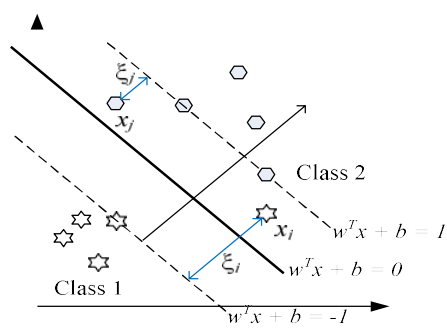

Figure 1. Slack variables for non-separable data

The unsatisfactory linear SVM performance could be replaced by the use of the nonlinear SVM algorithm. A higher-dimensional space is formed from a non-linear mapping $\varphi(x)$ derived from the value of $\mathrm{x}$, hence an optimal hyperplane is founded. The kernel function calculation $\mathrm{K}(\mathrm{xi}, \mathrm{xj})$ could implicitly map the inner product of vectors $\varphi(\mathrm{xi})$ and $\varphi(\mathrm{xj})$. The radial base function and polynomial function are respectively shown in equations (3) and (4).

$$
\begin{aligned}
& \mathrm{K}\left(\mathrm{x}_{\mathrm{i}}, \mathrm{x}_{\mathrm{j}}\right)=\exp \left(-0.5 \frac{\| \mathrm{x}_{\mathrm{i}-\mathrm{x}_{\mathrm{j}} \|^{2}}}{\sigma^{2}}\right) \\
& \mathrm{K}\left(\mathrm{x}_{\mathrm{i}}^{\mathrm{x}} \mathrm{x}_{\mathrm{j}}\right)=\left(\mathrm{x}_{\mathrm{i}}^{\mathrm{T}} \mathrm{X}_{\mathrm{j}}^{\mathrm{T}}+1\right)^{\mathrm{d}}
\end{aligned}
$$

Both equations are the typical kernel functions that are used for this problem. The class label SVM of sample $\mathrm{x}$ in a classification stage is expresed by the following decision function:

$$
f(x)=w^{T} \emptyset(x)+b=\sum_{i=1}^{N} \quad \alpha_{i} y_{i} K\left(x_{i}, x\right)+b
$$




\section{II.2. One-Against All (OAA) Method}

Consider an $\mathrm{n}$ training samples: $\left\{x_{1}, y_{1}\right\}, \ldots,\left\{x_{n}, y_{n}\right\}$ in m-class problem. Here $y i \in\{1,2, \ldots, m\}$ is the corresponding label of class, and $x_{i} \in R^{m}$ is an m-dimensional feature vector. OAA method develops $\mathrm{m}$ binary classifiers of SVM, which divides one class from all the rest. In this method, the $i$ th class with positive labels is trained with the rest of the training examples, and all the others with negative labels. The following problem that generates the $i$ th decision function $f_{i}(x)=w^{T} \varphi_{i}(x)+b_{i}$, mathematically solved by

the $i$ th SVM.

minimize:

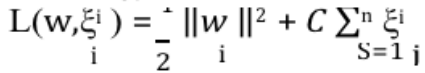

subject to:

$\bar{y}_{i}\left(w_{i}^{T} \phi\left(x_{i}\right)+b_{i}\right) \geq 1-\xi_{\mathbf{j}}^{i}, \xi_{j}^{i} \geq 0$

where

$\tilde{y}_{\mathrm{j}}=1$ if $y_{j}=i$ and $\tilde{y}_{\mathrm{j}}=-1$ otherwise.

At the classification stage, a sample $x$ is classified as in class $i^{*}$ whose $f_{i *}$ yields the largest value $\mathrm{i}^{*}=\arg \max \mathrm{f}_{\mathrm{i}}(\mathrm{x})=\arg \max \left(\mathrm{w}^{\mathrm{I}} \emptyset(\mathrm{x})+\mathrm{b}_{\mathrm{i}}\right)$

$$
\mathrm{i}=1, \cdots, \mathrm{m} \quad \mathrm{i}=1, \cdots, \mathrm{m} \quad \mathrm{i}
$$

\section{RESEARCH METHODOLOGY}

\section{III.1. The Measurement of Bilateral PPG}

In this research, the system is built to record and monitor the changes in blood volume between the right and the left thumb side on hand. To obtain a reference signal from the PPG probe, two clips are attached to the thumbs of the right and left hands, as shown in Figure 2 (a). With a sampling rate of $1 \mathrm{kHz}$, bilateral PPG is obtained synchronously through the MSP430 embedded two-channel measurement system. The data generated by the analog to the digital part of the MSP430 is transferred to a laptop for further analysis using MATLAB 2014a. By using a relatively low voltage source of 5 volts, the module is shown in Figure 2 (b) can be a continuous and promising measurement tool during hemodialysis treatment.

(a)

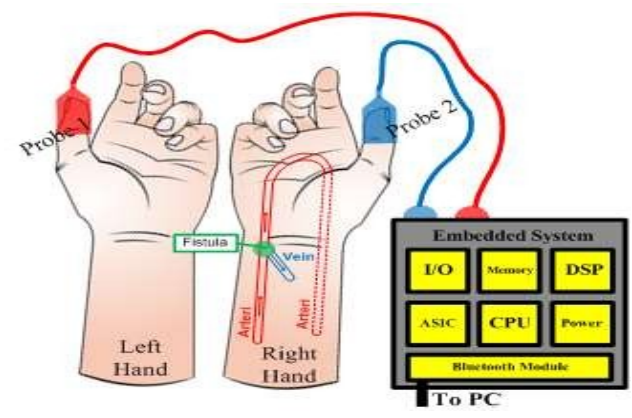

(b)

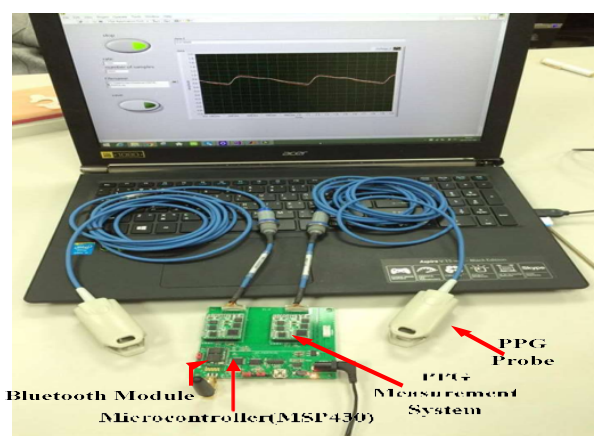

Figure 2. (a) The site PPG probe mounted and (b) The prototype of the system. 


\section{III.1. Experiment to detect arteriovenous fistula (AVF) stenosis}

In this experiment, 22 subjects with a mean age of $69.4 \pm 13.1$ years were recruited from the Kaohsiung Veterans General Hospital (KVGH), Tainan Branch - Institutional Review Board (IRB), under the number of contracts: VGHKS13-CT12 -11. Seven data sets were composed for the test model, 15 sets were randomly chosen to validate the proposed training model. These subjects were divided according to its Degree of Stenosis (DoS) severe class. DoS, in the terminology of clinical research, is the degree of narrowing normal vessels in HD patients who have had vascular access (AVF). DoS is obtained from the measurement of angiography images or B-mode ultrasound by medical experts who follow the IRB approval procedure. DoS expressed in Equation (8), where D expresses the normal vessel diameter in the direction of blood flow, and d expresses the stenosis lesion diameter (Wu et.al, 2015).

$$
\operatorname{DoS} \%=\left(\frac{\mathrm{d}^{2}}{\mathrm{n}^{2}}\right) \times 100 \%
$$

Total occlusion is indicated by a DoS value of $100 \%$. If the DoS value is greater than $50 \%$, then surgical treatment is required. When the DoS value is between $30 \%-50 \%$, it means there may be an efficiency effect during HD treatment. Thus, in this study to classify three different degrees of stenosis, clinical comments were used, as shown in Table 1.

Table 1

DoS based class partition

\begin{tabular}{cc}
\hline Class & DoS \\
\hline 3 & $D o S \geq 50 \%$ \\
2 & $30 \% \leq D o S \leq 50 \%$ \\
1 & $D o S \leq 30 \%$ \\
\hline
\end{tabular}

AVS Stenosis Detecting Algorithm

To detect AVF stenosis according to its degree of stenosis severe class, the bilateral differences from the PPG signal acquired has to be calculated to extract the feature pattern of blood volume changes in the time domain. Based on this bilateral PPG signal, a technique to detect AVF stenosis was built, which procedure is shown in Figure 3.

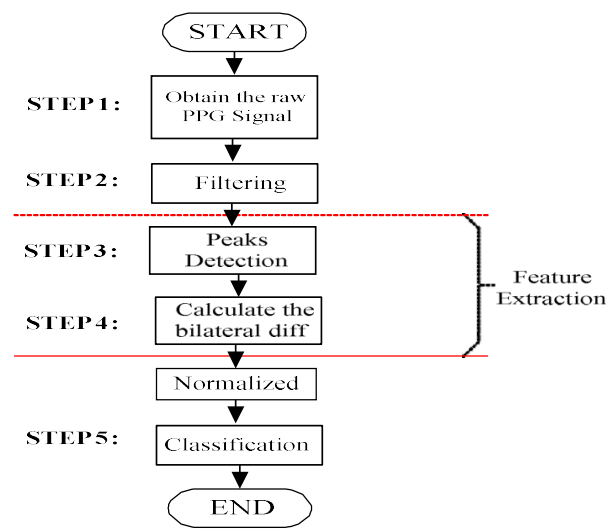

Figure 3. Procedure of AVF stenosis detection flowchart The research procedure is described as follows: 
Step 1) Obtain a raw PPG signal from a subject before HD process, the measurement embedded system.

Step 2) Preprocess the acquired signal, before extracting process the raw PPG signal needs to be filtered to get a smooth PPG signal using a moving average filter, and the length of 2000 elements has been used.

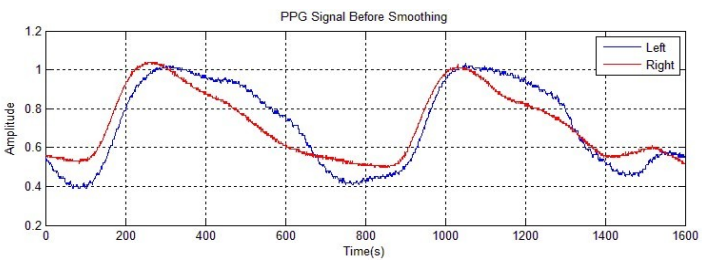

(a)

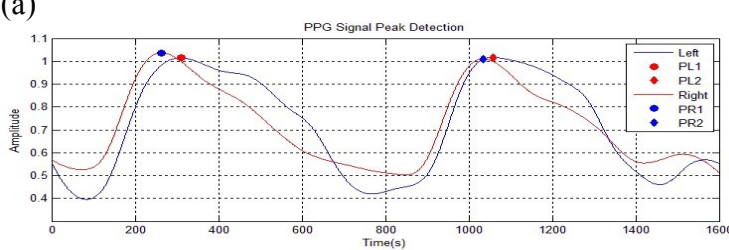

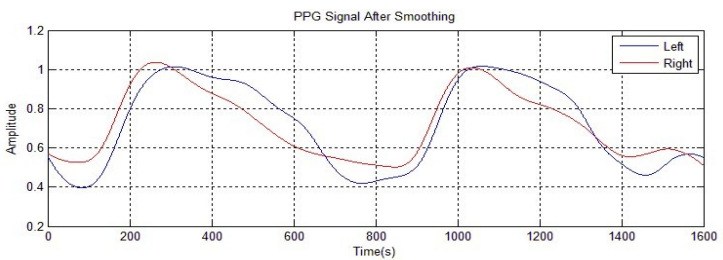

(b)

(c)

Fig. 4. (a) - (c) Step 1 - Step 3 Process

Step 3) Finding two peaks of the filtered signal. To detect each peak of the signal, local maxima algorithm being used. Data of PPG row vector with real-valued elements with a minimum length of three compares neighboring values to its data element. If the data element equal to infinity or larger than both of its neighbors, the element is a local peak.

Step 4) Calculate its rising time to reach the peak ( $R T=|R T R-R T L|)$. Next, we normalized this data with the highest result data. Step 3 and 4 was a Feature Extraction procedure for this proposed algorithm. This procedure was repeated to get the feature of the Bilateral PPG signal after the hemodialysis (HD) process.

Step 5) Finally, based on the subject with and without the vascular disease, the subject was divided into three groups considering the DoS of each subject : Normal Subject $(n=8)$, Low Grade Subject $(n=8)$ and High Grade Subject $(n=6)$. In this study we used STPRTOOL (Statistical Pattern Recognition Toolbox for MATLAB)[14] for doing classification with SVM OAA as multiclass method.

Data training and testing are stages that generally exist in a classification process. The instance in the training set consists of one class label and several attributes. The goal of SVM OAA is to generate a model that could predict the target value of data instances in the testing set, which is given only the attribute.

\section{FINDING AND DISCUSSION}




\section{IV.1. Feature extraction of Bilateral PPG}

In this experimental design, two clips of probes were used for non-invasive measurement, which operates in reflectance mode at the skin surface. The moving average filter could smooth the signal and keep the shape without losing the information. The local maxima algorithm is then being used to detect the peak of each signal, as shown in Figure 5. And the location of the peak then considers its rise time. Furthermore, Bilateral differences being calculated before and after the HD process to built a feature pattern. These patterns divided into three groups according to the DoS-severe.
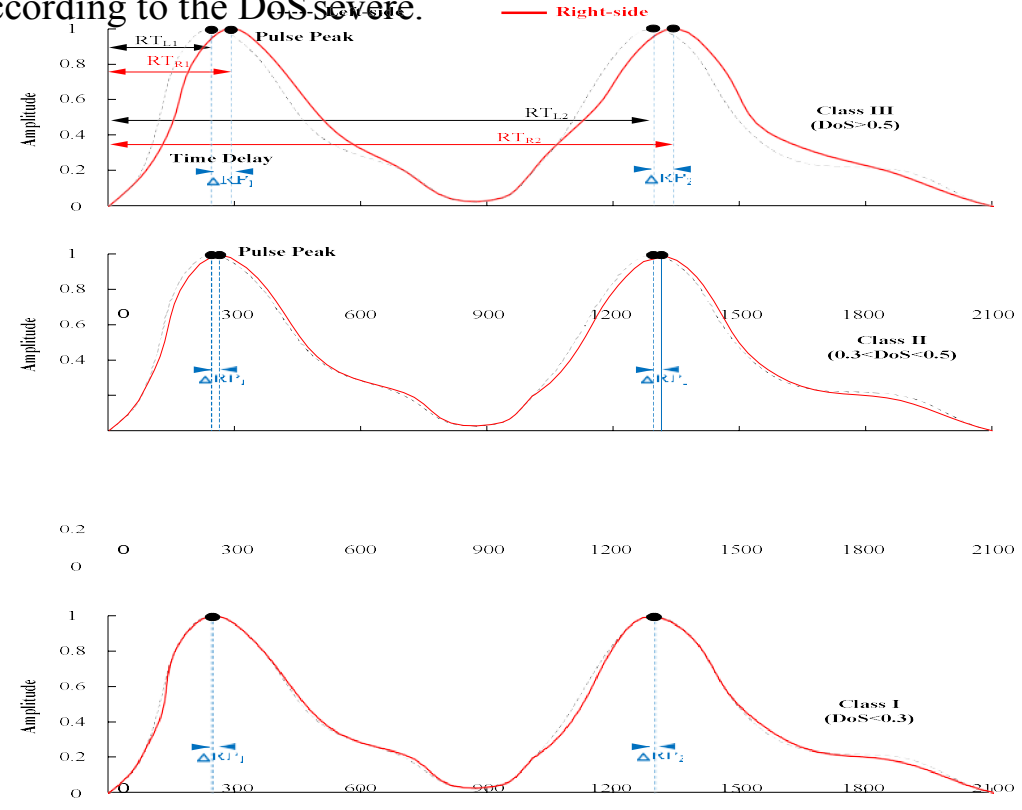

Fig. 5. Local maxima algorithm to detect PPG signal's peak.

\section{IV.2. Performance Evaluation of Detecting AVF Stenosis}

We applied the aforementioned multiclass SVM OAA method on Bilateral PPG signals, there for the signal itself is divided into $m$ classes of $m$ binary classifiers. Our aim is to optimize the classifier performance and to reach a higher percentage in the accuracy. In this work, a 5-fold cross-validation algorithm was used to objectively evaluate the entire dataset and finally, regularization parameter $\mathrm{C}=30$ and $\sigma=1,5$ to determine the effective width of the RBF kernel was selected. The training data and the decision boundary is visualized in Figure 6. 
Fig. 6. Multiclass SVM classifier build by OAA decomposition.

The confusion matrix was built on stage of performance evaluation, which used 15 subjects data to train the system. For the purpose of testing each fold, seven randomly selected patient PPG Bilateral signal data were used. Table 2 shows the confusion matrix that drives the results of the proposed algorithm. According to the results shown in Table 2, the proposed technique gives a satisfactory conclusion with an overall $85.7 \%$ accuracy rate. While if considered the precision (Positive Prediction Value - PPV) and the sensitivity (Recall), the proposed method reaches $89.0 \%$ and $83.33 \%$ respectively. This system is consuming just 0.273 seconds for training. The experimental testing stage result shows the high performance of the proposed detecting AVF stenosis technique.

Table 2

Confusion Matrix Result

\begin{tabular}{|c|c|c|c|c|c|}
\hline \multirow{2}{*}{\multicolumn{2}{|c|}{$\begin{array}{c}\text { Accuracy = } \\
0.857\end{array}$}} & \multicolumn{3}{|c|}{ Prediction } & \multirow{2}{*}{$\begin{array}{c}\text { Class } \\
\text { Recall }\end{array}$} \\
\hline & & 1 & 2 & 3 & \\
\hline \multirow{3}{*}{ 焉 } & I & 3 & 0 & 0 & I \\
\hline & 2 & 0 & 2 & 0 & l \\
\hline & 3 & 0 & i & 1 & 0.5 \\
\hline \multicolumn{2}{|c|}{ Class Precision } & $\overline{1}$ & 0.6 & 1 & \\
\hline
\end{tabular}

\section{CONCLUSION AND FURTHERRESEARCH}

This research proposed a noninvasive technique based on the bilateral PPG signal for monitoring and detect the arteriovenous fistula stenosis. The equipment here was designed to record and collect the blood volume changes data wirelessly for further analysis on a laptop. Based on the experimental results, the technique used in this study can extract features effectively bilateral PPG signals to detect AVF stenosis in a research subject. The confusion matrix used as a classifier performance measurement tool shows high-performance results in identifying AVF stenosis via PPG signals.

\section{ACKNOWLEDGEMENTS}

The author would like to thank Dr. Ming-Jui Wu and Associate Professor Yi-Chun Du, Ph.D for their helpful comments, suggestions, and research data providing. 


\section{REFERENCES}

National Kidney Foundation, 2006. 2006 updates clinical practice guidelines and recommendations.

Vesquez, P.O., Marco, M.M. and Mandersson, B., 2009, September. Arteriovenous fistula stenosis detection using wavelets and support vector machines. In 2009 Annual International Conference of the IEEE Engineering in Medicine and Biology Society (pp. 1298-1301). IEEE.

Wu, J.X., Lin, C.H., Du, Y.C., Chen, P.J., Shih, C.C. and Chen, T., 2015, October. Estimation of arteriovenous fistula stenosis by FPGA based Doppler flow imaging system. In 2015 IEEE International Ultrasonics Symposium (IUS) (pp. 1-4). IEEE.

Wu, J.X., Lin, C.H., Du, Y.C. and Chen, T., 2012. Sprott chaos synchronisation classifier for diabetic foot peripheral vascular occlusive disease estimation. IET Science, Measurement \& Technology, 6(6), pp.533-540.

Allen, J., 2007. Photoplethysmography and its application in clinical physiological measurement. Physiological measurement, 28(3), p.R1.

Bash, S., Villablanca, J.P., Jahan, R., Duckwiler, G., Tillis, M., Kidwell, C., Saver, J. and Sayre, J., 2005. Intracranial vascular stenosis and occlusive disease: evaluation with CT angiography, MR angiography, and digital subtraction angiography. American journal of neuroradiology, 26(5), pp.1012-1021.

Tessitore, N., Bedogna, V., Gammaro, L., Lipari, G., Poli, A., Baggio, E., Firpo, M., Morana, G., Mansueto, G. and Maschio, G., 2003. Diagnostic accuracy of ultrasound dilution access blood flow measurement in detecting stenosis and predicting thrombosis in native forearm arteriovenous fistulae for hemodialysis. American journal of kidney diseases, 42(2), pp.331341.

Wang, H.Y., Wu, C.H., Chen, C.Y. and Lin, B.S., 2014. Novel noninvasive approach for detecting arteriovenous fistula stenosis. IEEE Transactions on biomedical engineering, 61(6), pp.18511857.

Wu, J.X., Lin, C.H., Wu, M.J., Li, C.M., Lim, B.Y. and Du, Y.C., 2015. Bilateral photoplethysmography analysis for arteriovenous fistula dysfunction screening with fractionalorder feature and cooperative game-based embedded detector. Healthcare technology letters, 2(3), pp.64-69.

Leon, C. and Asif, A., 2007. Arteriovenous access and hand pain: the distal hypoperfusion ischemic syndrome. Clinical journal of the American Society of Nephrology, 2(1), pp.175-183.

Wu, J.X., Li, C.M., Ho, Y.R., Wu, M.J., Huang, P.T. and Lin, C.H., 2015. Bilateral photoplethysmography analysis for peripheral arterial stenosis screening with a fractional-order integrator and info-gap decision-making. IEEE Sensors Journal, 16(8), pp.2691-2700.

Cortes, C. and Vapnik, V., 1995. Support-vector networks. Machine learning, 20(3), pp.273-297.

Wu, J.X., Lin, C.H., Du, Y.C., Chen, P.J., Shih, C.C. and Chen, T., 2015, October. Estimation of arteriovenous fistula stenosis by FPGA based Doppler flow imaging system. In 2015 IEEE International Ultrasonics Symposium (IUS) (pp. 1-4). IEEE.

Franc, V. and Hlavác, V., 2004. Statistical pattern recognition toolbox for Matlab. Prague, Czech: Center for Machine Perception, Czech Technical University. 